\title{
Application Conception of the Application of VR Technology in the Book Interview of University Library
}

\author{
Qian $\mathrm{Xu}^{1, \mathrm{a}}$
}

${ }^{1}$ East China Jiaotong University, Nanchang, Jiangxi, China, 330013

1093404970@qq.com

\begin{abstract}
Along with the development of science and technology, a new round of technological innovation, among which, most people expect, is the use of VR technology. VR used in people's daily life is also used in learning life. This paper will take the application of VR in university library as an example, reflect the current situation of the application of VR technology in real life, compare the traditional library interview mode, analyze the advantages and disadvantages of the application of VR technology, and analyze the problems arising from the application process of changing technology, and put forward policy suggestions to make up for the deficiency. Keywords: VR technology; libraries; present situation
\end{abstract}

\section{VR技术应用在大学图书馆图书采访中的应用构想}

\author{
徐茜 ${ }^{1, a}$
}

'华东交通大学, 南昌, 江西, 中国, 330013

a1093404970@qq.com

\section{摘要}

随着科技的发展，新一轮的技术革新产生，其中，最为人们所期待的，便是VR技术的运用。VR运用在 人们的日常生活中也运用在学习生活中。本文将以VR在大学图书馆的运用为例, 反应VR技术在现实生 活中的运用现状，对比传统的图书馆采访模式，分析VR技术运用的优点和不足，并分析改技术在运用 过程产生的问题，提出政策建议以弥补不足。

关键词: VR技术; 图书馆; 现状

\section{VR技术的使用现状研究}

作为当今世界的前沿科技之一，世界各国都纷纷 对VR技术表现出浓厚的兴趣, 各大公司先后加入VR产 品的研发当中去, 例如三星、亚马逊、索尼、华为等 公司, 都想通过研发率先抢占市场。VR技术的运用领 域广泛, 就目前而言, 主要是在现场直播、房地产展 示以及电子游戏等领域运用，身临其境的感觉，给使 用者带来了良好的使用体验。除此之外, VR技术还有 可以运用于远程医疗、工程仿真等领域。VR技术在这 些领域的运用不仅可以解决了这些领域之前存在的一 些问题而且还可以提高工作效率。2018年VR技术在昌 平冬奥会上的使用效果良好, 让人们看到了VR技术的 价值，推动了VR产业的发展。

除了各大企业发现VR技术未来的巨大运用潜力外, 国内外的各大图书馆也瞄上了VR技术, 纷纷决定在各 自的图书馆中使用该项技术。例如美国某实验室, 推 出了VR使用技术的客户端程序。不甘落后的其他图书
馆也开始纷纷将VR技术运用在自己的图书馆中，开展 虚拟图书馆业务。当人们走进这样一所图书馆中，不 仅能够感受到书墨的芬香, 还能够感受到科技的鬼未 以及身临其境的体验。尽管如此, VR技术仍然未被充 分挖掘, 还有巨大的挖掘空间, 目前VR技术的主要运 用也只在信息检索服务、漫游服务、虚拟空间等领域。

\section{VR技术运用于图书采访中}

作为一种重要的创新方式, VR技术可以运用在图 书采访的众多方面，这其中就包括中标书目挑选和VR 技术实现虚拟现场采购。在这两个方面, VR技术能都 发挥出巨大的作用。同时通过在这两方面的讲价分析, 也能多众多运用VR技术方面提供帮助，以下就是具体 说明，希望通过这种构想，应在在图书采访中能有有 积极有效的作用。 
表1 V R 技术运用于图书采访中

\begin{tabular}{|c|c|c|c|}
\hline $\begin{array}{c}\text { 荐购信息收 } \\
\text { 集 }\end{array}$ & $\begin{array}{c}\text { 中标书目挑 } \\
\text { 选 }\end{array}$ & $\begin{array}{l}\text { VR技术实现 } \\
\text { 虚拟现场采 } \\
\text { 购 }\end{array}$ & $\begin{array}{c}\text { 新书预读预 } \\
\text { 热 }\end{array}$ \\
\hline $\begin{array}{l}\text { 使用VR技术 } \\
\text { 可以帮助采 } \\
\text { 访者更全面 } \\
\text { 且迅速地抓 } \\
\text { 住读者的需 } \\
\text { 求, 利用 } R \\
\text { 技术构建虚 } \\
\text { 并构建一个馆, } \\
\text { 虚拟教师会 } \\
\text { 议室、读者 } \\
\text { 题讨论、主 } \\
\text { 学 } \\
\text { 域、本前沿推 } \\
\text { 荐区等等, } \\
\text { 让更多读者 } \\
\text { 畅所欲言, } \\
\text { 棵题、特 } \\
\text { 色资源、科 } \\
\text { 研前沿、图 } \\
\text { 书荐购等话 } \\
\text { 题的讨论 }\end{array}$ & $\begin{array}{l}\text { 读者可以根 } \\
\text { 据自身的选 } \\
\text { 择, 进行书 } \\
\text { 䉈地阅读, } \\
\text { 其阅读的体 } \\
\text { 验与真实 } \\
\text { 的读书场景 } \\
\text { 体 验感无 } \\
\text { 差, 并且通 } \\
\text { 过 VR 技术 } \\
\text { 采, 访人员 } \\
\text { 还可以随时 } \\
\text { 的与读者进 } \\
\text { 行交流, 这 } \\
\text { 便增加了读 } \\
\text { 书互动感, } \\
\text { 使读者有更 } \\
\text { 的訚。读体 }\end{array}$ & $\begin{array}{l}\text { VR技术运用 } \\
\text { 于采购主要 } \\
\text { 是通过模拟 } \\
\text { 虚拟的采购 } \\
\text { 景, 设置 } \\
\text { 虚拟货架, } \\
\text { 信息通过VR } \\
\text { 技术步到 } \\
\text { 体验者那 } \\
\text { 里, 就像现 } \\
\text { 场选书一 } \\
\text { 样, 体验者 } \\
\text { 能够直观的 } \\
\text { 感受到书籍 } \\
\text { 的外观以及 } \\
\text { 主要 简 介 }\end{array}$ & $\begin{array}{l}\text { 使 用 VR 技 } \\
\text { 术, 供应商 } \\
\text { 在签订购买 } \\
\text { 协议时, 需 } \\
\text { 要将图书的 } \\
V R \text { 资源提前 } \\
\text { 送到图 书 } \\
\text { 馆。在纸质 } \\
\text { 书籍处理之 } \\
\text { 前, 图馆 } \\
\text { 将书籍的虚 } \\
\text { 拟现实资源 } \\
\text { 发布到虚拟 } \\
\text { 现实虚拟社 } \\
\text { 方便读 } \\
\text { 者找到自己 } \\
\text { 感兴趣的书籍。 }\end{array}$ \\
\hline
\end{tabular}

\section{1 荐购信息收集}

读者需求是文献采购的重要依据, 但读者需求是 最难把握的。在传统的采访模式下, 采访者通过对各 高校的实地调研, 掌握教师教学和科研文献的需求。 然而, 由于时间和地点的限制, 并不是所有的教师都 能参与, 收集的信息往往不够充分。采访者利用互联 网通过读者的网上留言、微信、电子邮件、图书荐购 系统等收集读者信息, 因而无法与读者直接互动、及 时反馈, 导致读者荐购热情不高, 只能收到零星的购 书建议, 无法充分把握读者的真实需求。

使用VR技术可以帮助采访者更全面且迅速地抓住 读者的需求, 利用 $\mathrm{R}$ 技术构建虚拟图书馆, 并构建一个 虚拟教师会议室、读者讨论区、主题讨论、学科前沿 领域、本书推荐区等等, 让更多读者畅所欲言, 积极参 与热门课题、特色资源、科研前沿、图书荐购等话题 的讨论, 将相关评论呈现在VR虚拟社区场景中, 采访 人员可随时查看并参与讨论, 快速掌握学科、科研信 息和读者需求, 进而完善馆藏资源的配置。

\section{2 中标书目挑选}

VR技术在图书馆的使用主要是为了实现在选书过 程中读者与书有较高的交互性。当读者进入图书馆的
VR场景中，便如同置身于真实的商店中，但是书籍的 搜索又比真实的商店便捷。同时每本书除了有基本信 息外，还附带着音频等资源。读者可以根据自身的选 择, 进行书籍地阅读, 其阅读的体验感与真实的读书 场景体验感无差, 并且通过VR技术采, 访人员还可以 随时的与读者进行交流, 这便增加了读书互动感, 使 读者有更好的阅读体验。

\section{$2.3 V R$ 芰术实现虚拟现场采购}

利用VR技术实现虚拟现场采购。VR技术运用于采 购主要是通过模拟虚拟的采购场景, 设置虚拟货架, 将每本书的信息通过VR技术同步到体验者那里, 就像 现场选书一样, 体验者能够直观的感受到书籍的外观 以及主要简介等。这项技术不受时间与空间的限制, 方便了人们的远距离挑选，同时还可以直接与销售人 员互动, 并且通过互联网直接对信息的采集与录入, 这样不仅降低了人们采购所需的时间, 也减少了为去 采购而花费的时间, 到达减少采购成本的目的。与此 同时，此项技术还能提高在图书购买过程中效率。

\section{4 新书预读预热}

使用VR技术，供应商在签订购买协议时，需要将 图书的VR资源提前送到图书馆。在纸质书籍处理之前, 图书馆将书籍的虚拟现实资源发布到虚拟现实虚拟社 区，方便读者找到自己感兴趣的书籍。首次阅读新书 时, 读者还可以在VR虚拟社区中撰写新书评论并进行 展示, 引起各网友的注意, 激发读者的阅读兴趣, 促 进新书的推广，提高新书的利用率。

\section{3. 在运用 V R 技术于图书馆图书采访中存在 的问题}

因为是新兴技术，其运用在图书馆采访中的过程 中, 一定会出现多种问题, 以下就是相关问题:

\section{1 人员的关注不够}

虽然国家发布了许多关于布局前沿技术的政策， 但是普通民众对这些前沿性的科学技术了解并不多, 甚至一点了解都没有, 根本不知道是什么。并且相对 于人工智能等领域, 知道 $\mathrm{VR}$ 技术的人们反而更少。就 此而言, 人们难以认识到图书馆运用VR技术的好处。 其次, VR技术虽然是相对成熟, 但是却不是一个完美 的技术, 它还存在许多的创新进步空间, 以便能运用 到更多领域, 同时也应该实现这项技术的工业化, 做 到白菜价。但是由于对这些技术的关注不够，研发投 入也相对较少, 目前VR技术还要继续发展。 


\subsection{VR 发展缺乏资源}

虽然VR发展有了一定的基础也到了一定的阶段， 但是VR在图书中的运用, 还存在着许多问题, 就目前 来说, VR图书和书目业并没有形成很好的行业标准体 系，制作成本过高是导致VR发展资源极度贵乏的一个 重要原因。在这样的情况下, 难以形成一个完善的体 系, 就无法满足批量的采访。一方面, 没有书商制作 商等提供书目, 也没有行业标准, VR选书虚拟现场采 购难以开展。另一方面, 需要专业的团队、大量的资 金投入、大量的时间才能完成2D资源的3D资源的转换。 出于盈利考虑, 很多出版商, 制作商都不愿意投入。 在没有行业标准又缺乏投资的情况下, VR图书是无法 批量生产的。

\section{3 用户接受程度不高}

目前VR技术正在完善中，在发展过程中，体现出 很多问题: 性能差、舒适度低、用户体验不好, 设备 价格昂贵。因此, 大部分人无法接受。通过VR, 用户 可以身临其境, 以计算机技术为核心的现代高科技手 段为载体, 自然地和虚拟世界中的对象进行互动。这 一过程通常需要VR眼镜来实现, 但是性能高、舒适度 高的VR眼镜价格很高。此外, VR眼镜的使用也不是很 舒服，有眩晕感基于以上原因，会导致用户体验不佳， 从而导致接受程度不高。

\section{V R 技术在图书采访中的应用对策}

VR技术如何应用在图书采访过程中呢? 最主要有 以下几种对策, 通过多种渠道, 多种方式, 总结出 “V R +采访” 准确定位, 需求驱动行业技术革新, 模式推广，三种对策，具体描述如下:

\section{表2 VR技术在图书采访中的应用对策}

\begin{tabular}{|c|c|c|c|}
\hline $\begin{array}{c}\text { “V R+采 } \\
\text { 访” 准确定 } \\
\text { 位 }\end{array}$ & $\begin{array}{c}\text { 完善标准化 } \\
\text { 体系 }\end{array}$ & $\begin{array}{l}\text { 需求驱动行 } \\
\text { 业技术革新 }\end{array}$ & VR模式推广 \\
\hline "V V R + 采 & 图书馆应与 & 通过需求促 & 组建科研团 \\
\hline 访” 不仅是 & 相关部门共 & 进供给, 让 & 队进行研 \\
\hline 一种新的采 & 同努力, 积 & VR资源逐渐 & 究, 然后加 \\
\hline 访形式, 应 & 极推动 VR 图 & 多起来, 资 & 以完善; 在 \\
\hline 该意味着有 & 书和VR书目 & 源体系逐渐 & 设备方面， \\
\hline 更高的采访 & 标准体系的 & 完善, VR的 & 要不断增加 \\
\hline 质量, 更好 & 制定，这必 & 门槛降低， & VR设备设施 \\
\hline 的配 置资源 & 将推动整个 & 读者所能接 & 资源 的 购 \\
\hline 以及更好地 & VR行业的快 & 触的资源就 & 置; 要加强 \\
\hline 掌握读者的 & 速发展。VR & 会更多, 接 & 图书管理员 \\
\hline 需求, 然后 & 行业标准体 & 受程度也会 & 的技术培 \\
\hline 最大限度的 & 系的建立, & 慢慢变高。 & 训, 让他们 \\
\hline 配置图书资 & 将 使 VR 厂 & & 掌握软硬件 \\
\hline 源在采访中 & 商、出版商 & & 的使用 方 \\
\hline 的应用, 是 & 和书商按照 & & 法; 为方便 \\
\hline 图书馆应用 & 统一标准进 & & 读者, 要更 \\
\hline 中 的一 部 & 行生产, 资 & & 好地打造虚 \\
\hline
\end{tabular}

\begin{tabular}{|l|l|l|l|}
\hline 分。 & 源和技术的 & 拟社区, 打 \\
& 交流, 加强 & & 造读者交互 \\
& 合作, 增强 & & 平台。 \\
VR 图书和VR & & \\
& 书目在各种 & & \\
& 收购平台上 & & \\
& 的适用性。 & & \\
\hline
\end{tabular}

\section{1 “ $V R$ +采访” 准确定位}

“V R +采访” 不仅是一种新的采访形式, 应该 意味着有更高的采访质量, 更好的配置资源以及更好 地掌握读者的需求, 然后最大限度的配置图书资源在 采访中的应用, 是图书馆应用中的一部分。图书馆要 重视VR技术的发展, 可以加大这个模式的投入，从而 更好地运用好VR技术。

\section{2 完善标准化体系}

图书馆应与相关部门共同努力, 积极推动VR图书 和VR书目标准体系的制定，这必将推动整个VR行业的 快速发展。VR行业标准体系的建立, 将使VR厂商、出 版商和书商按照统一标准进行生产, 资源和技术的交 流, 加强合作, 增强VR图书和VR书目在各种收购平台 上的适用性。该标准体系的建立将有助于制造商、出 版商和书商解决VR应用中存在的问题。VR标准体系的 建立将降低VR应用成本, 降低VR图书、VR书目等产品 的生产成本。实现标准化生产将大大提高图书市场 VR 图书资源的供给。同时可以要求书商提供标准化的VR 书目，为图书采集提供VR图书资源和VR书目数据支持。 高校图书馆作为重要的图书消费市场, 也应该拥有一 定的话语权, 参与VR行业标准的制定, 使图书VR数据 和VR产品更适合高校图书馆的需要。

\section{3 需求驱动行业技术革新}

图书馆应该将虚拟资源的建设重视起来，作为此 项资源技术的需求方, 可以通过提高经费占比, 也可 以在招标文件中也可以明确指出优先选择VR资源, 通 过需求促进供给, 让VR资源逐渐多起来, 资源体系逐 渐完善, VR的门槛降低, 读者所能接触的资源就会更 多, 接受程度也会慢慢变高。

\section{4. $4 V R$ 模式推广}

图书馆通过以下几个方面可以进行宏观布局, 改 善构建 “ V R +采访” 的环境和条件。首先, 组建科 研团队进行研究, 然后加以完善。其次, 在设备方面, 要不断增加VR设备设施资源的购置。再有, 要加强图 书管理员的技术培训，让他们掌握软硬件的使用方法。 以及, 图书馆界要和书商达成一致, 或者要求他们提 
供虚拟书目等。最后, 为方便读者, 要更好地打造虚 拟社区，打造读者交互平台。

\section{5. 结语}

作为一种当今社会的新兴技术, VR技术的产生在 当代的生活具有重要意义。同样, 在大学图书馆的图 书采访活动中, 一样有非常重要以及不可忽视的作用。 本文通过对VR技术在大学图书馆图书采访中的构想, 深刻分析了VR的应用可能性以及应用情况。希望以此 能给大学图书馆管理人员, 或者相关从事图书采访的 人员一点启示。

\section{REFERENCES}

[1] Weichao Li,Liping Bi,Haixia Zhao. Research on the Development of University Library Game Service in China[J]. Library Work and Research. 2017(4)

[2] Guoqiang Hu,Laihong Ma. Application of virtual reality and augmented reality in intelligent library[J]. Library Work and Research.2017

[3] Lihong Zhou,Yingying Han,Xiaomei Tu. Comparative Study on the Application of Virtual Reality Technology in University Libraries at Home and abroad $[\mathrm{J}]$. Books and intelligence.2017(4)

[4] Hebing,Hongyu Liu,Luonan. Research on University Library Information Service Based on
Virtual Knowledge Community[J]. Library Work and Research.2015(10)

[5] Hejian lu,Zhanghan. Reflections on Service Innovation by Using Virtual Reality Technology in Chinese and American Libraries[J]. Studies in Library Science. 2017(17)

[6] Yingjun Lu,Chenglei. A Study on Library Information Resource Construction and Service Innovation Based on Virtual Reality Technology -Taking CADAL as an Example[J]. Books and intelligence. 2017(4)

[7] Zhengwei, Zhangchao. The Design of a New Generation Library Management System Based on VR and AR Technology[J]. Computer Products and Circulation. 2019(11)

[8] Wenmin Zhai. Exploration on Service Thinking of College Library in VR Times[J]. Science and Technology Documentation Information Management. 2019(03)

[9] Luode Cheng, Fanyan, Wenge Fang, Zhangwei. Development and Application of VR Technology in Digital Library of Colleges and Universities[J] . Library science. 2019(01)

[10] Xiuli Wang, Yunmei Li. Exploration and Application of VR Technology in the Construction of University Library[J]. Agricultural network information. 2018(06) 